\title{
Future Time Perspective: Opportunities and Limitations Are Differentially Associated with Subjective Well-Being and Hair Cortisol Concentration
}

\author{
Pavel Kozik $^{a}$ Christiane A. Hoppmann ${ }^{a}$ Denis Gerstorf ${ }^{b}$

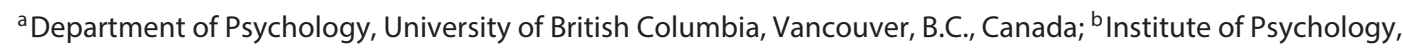 \\ Humboldt University Berlin, Berlin, Germany
}

\section{Key Words}

Future time perspective - Cortisol - Chronic stress .

Well-being

\begin{abstract}
Background: Future time perspective has been associated with subjective well-being, though depending on the line of research considered either an open-ended future time perspective or a limited future time perspective has been associated with high well-being. Most of this research however has conceptualized future time perspective as a one-dimensional construct, whereas recent evidence has demonstrated that there are likely at least two different underlying dimensions, a focus on opportunities and a focus on limitations. This project first seeks to replicate the two-dimensional structure of the Future Time Perspective Scale, and then examines the associations these dimensions may have with different measures of subjective well-being and a biological index of chronic stress. Objective: To test if the two dimensions of the Future Time Perspective Scale, a focus on opportunities and a focus on limitations, differentially associate with two measures of subjective well-being and a biological indicator of chronic stress, namely hair cortisol. Method: Sixty-six community-dwelling participants with a mean age of 72 years $(S D=5.83$ ) completed the Future Time Perspective Scale, Center for Epidemiologic Studies Depression Scale, and Philadelphia Geriatric Center Morale Scale. Participants
\end{abstract}

also provided a 3-cm-long hair strand to index cortisol accumulation over the past 3 months. Following the results of a factor analysis, a mediation model was created for each dimension of the Future Time Perspective Scale, and significance testing was done through a bootstrapping approach to harness maximal statistical power. Results: Factor analysis results replicated the two-dimensional structure of the Future Time Perspective Scale. Both dimensions were then found to have unique associations with well-being. Specifically, a high focus on opportunities was associated with fewer depressive symptoms and higher morale, whereas a low focus on limitations was associated with reduced hair cortisol, though this association was mediated by subjective well-being. Conclusion: Results replicate and extend previous research by pointing to the multi-dimensional nature of the Future Time Perspective Scale. While an open future time perspective was overall beneficial for well-being, the exact association each dimension had with well-being differed depending on whether subjective measures of well-being or biological indices of chronic stress were considered.

(c) 2014 S. Karger AG, Basel

\section{Introduction}

Future time perspective has a rich history within psychology [1]. Typically, individuals are thought to fall somewhere along a continuum that ranges from an open-

\section{KARGER 125}

(c) 2014 S. Karger AG, Base

0304-324X/14/0612-0166\$39.50/0

E-Mail karger@karger.com

www.karger.com/ger 
ended to a limited future time perspective with implications for their subjective well-being $[2,3]$. For example, Socioemotional Selectivity Theory proposes that when future time is perceived as limited, goals and priorities are shifted to enhance well-being $[4,5]$. However, there are also a number of studies suggesting that an open-ended rather than a limited future time perspective may be associated with high well-being $[2,6]$. Importantly, both of these lines of research have examined future time perspective from the viewpoint that it is a one-dimensional construct, whereas a recent line of studies has demonstrated that this might not be true [7-9]. Specifically, Cate and John [7] found that across different data sets the Future Time Perspective Scale consistently displayed two dimensions, a focus on opportunities and a focus on limitations, and further studies since have found both of these dimensions to have unique associations with workplace characteristics $[8,9]$. However, while there is initial evidence showing two dimensions, no study has yet examined whether or not each may be differentially associated with measures of well-being in old age and if this may help explain why a limited future time perspective can be associated with both high and low well-being. Hence, our study first sought to replicate the two-dimensional structure of the Future Time Perspective Scale as reported by Cate and John [7], and then to test each dimension in relation to two different measures of subjective well-being and a biomarker of chronic stress, namely hair cortisol [10]. Because we were interested in how each dimension may be uniquely associated with well-being, the addition of a biological measure in conjunction with our subjective measures allowed for a multifaceted examination. To do so, 66 older adults with a mean age of 72 years $(S D=5.83)$ completed the Future Time Perspective Scale [3] and two psychological measures of well-being (or lack thereof), the Center for Epidemiologic Studies Depression Scale (CES-D) [11] and the Philadelphia Geriatric Center Morale Scale (PGCMS) [12]. Participants then provided a hair strand sample to index cortisol accumulation over a past period of 3 months $[10,13]$.

\section{Future Time Perspective and Well-Being}

Research on future time perspective and well-being has often been conducted under the framework of Socioemotional Selectivity Theory $[3,4,14]$. This theory proposes that future time perspective (rather than age) plays a key role in determining why and when an individual may adjust their goals and priorities [4]. Support

Future Time Perspective and Well-Being for this theory comes from numerous studies showing that different future time perspectives are associated with particular social preferences [14, 15]. For example, Lang and Carstensen [4] found that older adults with a limited future time perspective preferred social interactions with family members, whereas those with an openended future time perspective preferred social interactions with friends and acquaintances. Similar social preferences have been shown in individuals who by virtue of their health, concerns about terrorism, or other social constraints had limited future time perspectives [14-16]. Although earlier notions of Socioemotional Selectivity Theory do not specifically address associations between perceptions regarding future time and well-being per se, more recent work elaborates that ' [Socioemotional Selectivity Theory] maintains that emotional experience improves because people become increasingly motivated to pursue emotionally meaningful goals and thus invest psychological and social resources to optimize emotional well-being' [5, p. 29]. Following this notion, older adults who perceive their remaining future time as more limited may be expected to report higher levels of well-being.

In contrast, a more recent line of studies has demonstrated that an open future time perspective rather than a limited one is associated with high well-being $[2,6,17]$. For instance, Hicks and colleagues [2] found that individuals with an open-ended future time perspective reported higher positive affect and meaning in life compared to those with a limited future time perspective. Furthermore, Allemand and colleagues [17] found that participants with an open-ended future time perspective reported more positive affect, life satisfaction and optimism, while also reporting less negative affect and pessimism. Finally, some studies have reported that a limited future time perspective is associated with certain maladaptive outcomes, including loss of hope, higher depression, and overall lower well-being $[6,18]$.

Taken together, these two streams of research thus come to different conclusions as to whether an open-ended or a limited future time perspective promotes high well-being. One unifying theme across these different studies however is that future time perspective has typically been conceptualized as a unidimensional construct. This conceptualization of future time perspective however may be incorrect, as Cate and John [7] found that across different data sets future time perspective consistently emerged as a two-dimensional construct composed of the dimensions a focus on opportunities and a focus on limitations. Since then, further studies have found these 
two dimensions to have unique workplace associations, for instance individuals with a high focus on opportunities report more positively about their future work and view their occupational future as involving more possibilities $[8,9]$. To our knowledge however, no study has yet examined whether or not these two dimensions differentially associate with well-being, and in particular, if considering the separate influence of each dimension may help explain why a limited future time perspective is sometimes associated with high well-being and at other times with low well-being.

\section{The Present Study}

The objective of the present study was to replicate the two-dimensional structure of the Future Time Perspective Scale as reported by Cate and John [7] and to examine if the two dimensions, a focus on opportunities and a focus on limitations, differentially associate with well-being. One might speculate that if the perception of future time is constricted for the opportunities dimension, this may motivate an individual to focus and selectively invest resources into the few opportunities that remain, thereby maximizing well-being. In contrast, if the limitations dimension is constricted, the individual might perceive their remaining future as being riddled with obstacles and hardships, which in turn may undermine well-being. To test if such potential relations exist, after replicating a two-dimensional structure of the Future Time Perspective Scale we aimed to test the unique association each dimension has with two commonly used psychological measures of well-being (or lack thereof), namely the CESD [11] and the PGCMS [12].

Lastly, we wanted to extend previous research that has largely been based on self-report measures of well-being by also including a biological measure of chronic stress (or lack thereof), namely hair cortisol [10, 13, 19]. Cortisol is a widely studied hormone governed by the hypothalamic-pituitary-adrenal (HPA) axis which is related to several other biological systems including both the immune system and inflammatory processes. Measures of cortisol are frequently done through samples of saliva, urine, blood and, more recently, hair, with each measurement method having ramifications for how the respective readings have to be interpreted [20].

The amount of cortisol an individual secretes over a period of months and that is captured in hair cortisol has been shown to be tied to both psychological and biological sources of stress. For example, studies focus- ing on psychological features of chronic stress have found that unemployed individuals, individuals who suffer from chronic pain and caregivers all have higher concentrations of cortisol within their hair follicles relative to controls $[19,21,22]$. At the same time, hair cortisol also is higher when individuals are exposed to biological stressors. For example, endurance athletes have been found to have higher concentrations of hair cortisol, which is likely at least in part due to their unique fitness demands and physical conditioning [23]. It is thus important to recognize that hair cortisol may reflect both psychological and biological sources of stress that occurred over a past period of 3 months [10]. Indeed, both sources of stress need not always come together as evident in a recent review article by Staufenbiel et al. [24] which noted that subjective measures of stress and hair cortisol often did not correlate, likely because they were tapping into different experiences of stress and timespan measures.

In light of this, for the present study hair cortisol was selected over other cortisol collection methods as it is tied best to our psychological variables of interest. While samples of blood, saliva and other measurements are common, they may be better suited for paradigms seeking to capture acute cortisol secretion specific to that moment, hour or day [25]. Cortisol collected from hair strands however represents at least 3 months of accumulation and from this allows one to index chronic stress $[10,19]$. Under the assumption that our primary variable of interest, future time perspective, remains relatively stable over a period of several months, we chose hair cortisol as a similarly stable measure.

By including a biological stress index, we may thus assess if future time perspective not only influences subjective well-being but also is implicated in biological processes related to chronic stress. Further, our results are strengthened by having a biological index of well-being that is not subject to self-report biases, including but not limited to biases due to retrospective memory, social comparisons, or social desirability [26].

In summary, first, we aimed to replicate the findings of Cate and John [7] by demonstrating that the Future Time Perspective Scale is a multi-dimensional construct composed of two dimensions, a focus on opportunities and a focus on limitations. Second, we wanted to examine the association each dimension had with two commonly used measures of subjective well-being, the PGCMS and CES-D. Third and lastly, we examined whether or not these two dimensions were differentially associated with hair cortisol, a biological indicator of chronic stress. 


\section{Method}

\section{Participants}

Participants were recruited throughout the Vancouver metropolitan area via advertisements placed in print media, online resources and public locations such as community centers, libraries and hair salons. Interested individuals were then informed that the study was about healthy aging in Canadian populations, and in particular the association between well-being and a stress hormone known as cortisol. Individuals were screened for eligibility, which consisted of being at least 65 years old, able to read newspaper size font, having hair the length of at least $3 \mathrm{~cm}$ and willingness to donate a $3 \mathrm{~cm}$ hair sample for cortisol analysis.

In total, 69 participants (46 women) ranging in age from 65 to 91 years ( mean $=71.94$ years, $\mathrm{SD}=5.83$ ) completed the study. The majority of these participants identified themselves as either Caucasian (62\%) or Asian (36\%) with one participant identifying as another ethnicity (1\%). Roughly half of all participants (48.5\%) had postsecondary education, $25 \%$ had attended a professional or trades school, and $14.1 \%$ finished high school only. A third of participants were married, $27.3 \%$ divorced, $19.7 \%$ widowed, $13.6 \%$ single and $6.1 \%$ reported other. Most participants reported living alone (49.2\%), followed by living with one other person $(29.5 \%)$. A little over a quarter of participants (28.8\%) also reported having provided care to someone within the last 3 months.

The majority of participants $(98.5 \%)$ reported their health as being 'fair' to 'excellent'. The most commonly reported medical condition was high blood pressure $(\mathrm{n}=12)$, followed by osteoporosis $(n=7)$ and arthritis $(n=6)$. The most commonly reported medications were levothyroxine, commonly known as Synthroid $^{\circledR}(n=9)$, followed by calcium supplements $(n=4)$. Participants also completed the perceived stress scale, a commonly used scale to measure the perception of stress [27]. The mean sum score was $13.5(\mathrm{SD}=6.27$, range $=2.00-29.00)$, and was comparable to other studies examining older adults [27]. A verbal fluency task $($ mean $=12.94, \mathrm{SD}=3.50$, range $=5.00-23.00)$ revealed that the cognitive functioning of our sample was similar to that of other studies of older adults [28].

A total of 3 participants were excluded from data analysis. One participant who was identified as neither Caucasian nor Asian was excluded so that we may potentially consider the effects of ethnicity on our variables of interest; another 2 participants were excluded for incomplete data on either the Future Time Perspective Scale or the CES-D. In sum, the data of 66 participants were analyzed. All study measures and procedures were approved by the University of British Columbia Behavioral Research Ethics Board.

\section{Procedure}

After obtaining consent, a 2-hour session commenced in which participants provided background information and completed several scales; those of which are relevant to the current study are described below. Following completion of these scales, a hair strand sample was collected from the back of participants' heads to measure cortisol concentration. All sessions were conducted in a psychology lab at the University of British Columbia or at another public location such as a café depending on participants' preferences.

Future Time Perspective and Well-Being

\section{Measures}

Future Time Perspective

Participants completed the 10-item Future Time Perspective Scale [3]. Each item on the scale was accompanied by 5 response options ranging from ' 1 - very untrue' to ' 5 - very true', with higher responses signifying a more open future time perspective. Three of the items were reverse coded as per scale instructions. The mean future time perspective was $3.21(\mathrm{SD}=0.69$, range $=1.60-5.00)$ and had a Cronbach's alpha of 0.88 .

\section{Well-Being}

To measure well-being, participants first completed the 20-item version of the CES-D [11]. Each item on the scale was accompanied by 4 response options ranging from ' 1 - rarely or none of the time' to ' 4 - most or all of the time', with higher responses more indicative of depressive symptoms. As per standard practice, participant's responses were quantified into a sum score that could range from 0 to 60 . The mean sum score was $10.70(\mathrm{SD}=9.52$, range $=0.00$ 46.00). Cronbach's alpha was 0.88. Twelve participants scored above the CES-D clinical threshold for depression [29].

Following this, all participants completed the 17 -item version of the PGCMS [12]. Participants completed the scale such that each high morale response received a value of ' 1 ' and low morale response a value of ' 0 ', so that the total score ranges from $0-17$ with higher values indicating higher morale. The mean sum score of participants was $12.41(\mathrm{SD}=3.06$, range $=4.00-17.00)$, Cronbach's alpha 0.75 .

\section{Hair Cortisol}

Participant's hair strands were cut with scissors at a minimal length of $3 \mathrm{~cm}$ and as close as possible to the scalp at the back of the head. If more than this length was cut, only the $3 \mathrm{~cm}$ closest to the scalp were analyzed. All hair samples were then wrapped in aluminum foil and stored in a dry and dark cabinet until being mailed to Dr. Kirschbaum's lab at Dresden Technological University, Germany [10]. After arriving, the hair samples were dried, milled, and analyzed using standard assays. Hair cortisol values that were 2 standard deviations above the hair cortisol mean were set at a value of exactly 2 standard deviations above the mean. Following these preparatory steps, the mean concentration of cortisol was $25.77 \mathrm{pg} /$ $\mathrm{mg}(\mathrm{SD}=17.16$, range $=6.01-66.00 \mathrm{pg} / \mathrm{mg})$. Cortisol assays collected and processed in this manner by the Kirschbaum lab have an intra-assay and inter-assay coefficient of variance below 8\% [10].

\section{Control Variables}

Numerous variables have been shown to influence hair cortisol so as to provide for a meaningful interpretation of our main results, participants' sex [13], ethnicity [24], age [13], body mass index (BMI) [30], current medical diagnosis [31,32], whether or not hair was dyed and whether or not hair has permanent waves were recorded and controlled for $[13,19,33]$. All of this information was obtained through the questionnaires participants completed at the start of the study.

\section{Data Analysis}

As health conditions have been found to influence hair cortisol concentration [31, 32], a series of dummy variables were created for each of the most commonly reported medical conditions (high blood pressure, osteoporosis, and arthritis) and medications being taken (Synthroid ${ }^{\circledR}$ and calcium supplements) such that presence of 
Table 1. Means and standard deviations of the central study variables as well as their intercorrelations $(n=66)$

\begin{tabular}{llrrrrrrrrrrrrrrrr}
\hline & Mean & SD & 1 & 2 & 3 & 4 & 5 & 6 & 7 & 8 & 9 & 10 & 11 & 12 \\
\hline 1 & Future Time Perspective Scale & 3.21 & 0.69 & - & $0.95^{* *}$ & $0.75^{* *}$ & $0.44^{* *}$ & $-0.31^{*}$ & -0.16 & 0.15 & 0.07 & 0.03 & 0.07 & 0.12 & 0.02 \\
2 & Focus on opportunities & 3.29 & 0.77 & - & - & $0.49^{* *}$ & $0.39^{* *}$ & $-0.34^{* *}$ & -0.07 & 0.18 & 0.11 & 0.00 & 0.06 & 0.19 & -0.01 \\
3 & Focus on limitations & 3.02 & 0.85 & - & - & - & $0.38^{* *}$ & -0.12 & $-0.29^{*}$ & 0.05 & -0.02 & 0.08 & 0.07 & -0.07 & 0.09 \\
4 & PGCMS & 12.40 & 3.06 & - & - & - & - & $-0.43^{* *}$ & $-0.28^{*}$ & 0.03 & -0.13 & 0.11 & -0.06 & -0.17 & -0.08 \\
5 & CESD & 10.70 & 9.52 & - & - & - & - & - & -0.16 & -0.05 & -0.04 & 0.08 & 0.18 & -0.11 & $0.25^{*}$ \\
6 & Hair cortisol & 25.77 & 17.16 & - & - & - & - & - & - & -0.10 & 0.20 & -0.04 & 0.05 & $0.30^{*}$ & 0.18 \\
7 & Age & 71.94 & 5.83 & - & - & - & - & - & - & - & -0.09 & -0.19 & 0.03 & -0.07 & 0.05 \\
8 & BMI & 24.96 & 4.01 & - & - & - & - & - & - & - & - & -0.09 & 0.00 & $0.28^{*}$ & -0.10 \\
9 & Ethnicity & 0.38 & 0.49 & - & - & - & - & - & - & - & - & - & -0.08 & -0.08 & 0.14 \\
10 & Sex & 0.65 & 0.48 & - & - & - & - & - & - & - & - & - & - & $0.41^{* *}$ & 0.15 \\
11 & Hair dyed & 0.41 & 0.50 & - & - & - & - & - & - & - & - & - & - & - & 0.11 \\
12 & Hair wavy & 0.11 & 0.31 & - & - & - & - & - & - & - & - & - & - & - & - \\
\hline
\end{tabular}

Means, standard deviations and interrcorelations for all participants. Ethnicity was coded as a dummy variable with Asian assigned a value of 1 and Caucasian a value of 0; Sex was coded as a dummy variable such that females were assigned a value of 1 and males a value of 0 . Hair dye and wavy hair were both dummy coded variables such that presence of hair feature (wavy or dyed) resulted in a value of 1 and absence of hair feature resulted in a value of 0 . Higher values for the Future Time Perspective Scale indicate a more open future time perspective, higher PGCMS scores indicate greater morale, higher CES-D scores indicate more depressive symptoms.

${ }^{*} \mathrm{p}<0.05{ }^{* *} \mathrm{p}<0.01$.

condition or medication resulted in a value of 1 and absence a value of 0 . No correlation however was found between any medical condition or medication being taken and hair cortisol. Participants who reported having thyroid problems or diabetes, two conditions known to impact HPA axis functioning, were dummy coded in a similar manner as mentioned above, though no correlation was found to hair cortisol. Lastly, participants who reported having a condition related to cardiovascular health, such as high blood pressure, heart disease, past heart surgery or arrhythmia, were dummy coded such that presence of any of these symptoms or conditions resulted in a value of 1 and absence a value of 0 . No correlation was found however between this gauge of cardiovascular health and hair cortisol.

For all analysis reported in the results section, participant sex, ethnicity, age, BMI and whether or not hair was dyed or wavy were controlled for. Sex was dummy coded with females receiving a value of 1 and males a value of 0 . Ethnicity was dummy coded such that Asians received a value of 1 and Caucasians a value of 0 . A dummy variable was created for whether or not hair was dyed and another for whether or not hair was permanently wavy; presence of hair feature resulted in a value of 1 and absence a value of 0 . None of these variables except for whether or not hair was dyed had an association with hair cortisol $[\mathrm{r}(64)=0.30, \mathrm{p}<0.01]$. Lastly, the same overall pattern of reported results is found when all nonsignificant control variables are dropped.

Following this, an exploratory factor analysis with varimax rotation was conducted on the 10 items of the Future Time Perspective Scale. For a set number of dimensions to be extracted, their associated eigenvalue had to be greater than 1 [34]. A macro SPSS script written by O'Connor was then used for confirmatory factor analysis [35]. Through this script, a Monte Carlo simulation of 1,000 parallel data points was computed and a resulting 95th percentile eigenvalue calculated. For a significant two-factor solution to emerge, the raw data eigenvalue would have to surpass this resulting permutation eigenvalue.
Two dimensions, a focus on opportunities and a focus on limitations, are expected to emerge from these factor analyses. All items loading onto either dimension will then be grouped into a single mean that represents that dimension. Mediation models may then be constructed for both dimensions; through this we may test if a focus on opportunities or a focus on limitations is associated with hair cortisol and if this association is mediated by the subjective well-being measures, namely the CES-D and the PGCMS. Significance testing of both mediation models will be carried out through a bootstrapping approach as recommended by Preacher and Hayes [36] over the more traditional Baron and Kenny strategy [37]. Given our limited sample size, a bootstrapping approach allowed for maximal statistical power while still controlling for type I error. Further, by repeatedly sampling from the same data set, we were able to achieve an empirical approximation of the sampling distribution and may gauge the indirect effects of subjective well-being measures on hair cortisol. Lastly, when testing the association between the opportunities dimension and hair cortisol, the limitations dimension will be set as a control variable and similarly when testing the association between the limitations dimension and hair cortisol, the opportunities dimension will be set as a control variable.

\section{Results}

Table 1 provides a summary of intercorrelations, means and standard deviations for all main variables relevant to the current study. Notably, having a more open future time perspective was associated with higher morale as measured by the PGCMS and lower scores on the CES-D. A focus on opportunities was associated with 
Table 2. Two-factor solution of the Future Time Perspective Scale $(n=66)$

\begin{tabular}{lll}
\hline Future Time Perspective Scale items & $\begin{array}{l}\text { Factor 1: focus on } \\
\text { opportunities }\end{array}$ & $\begin{array}{l}\text { Factor 2: focus on } \\
\text { limitations }\end{array}$ \\
\hline $\begin{array}{ll}\text { Varimax rotated factor analysis of Future Time Perspective Scale } \\
\text { I expect that I will set many new goals in the future }\end{array}$ & $\mathbf{0 . 8 2}$ & 0.13 \\
My future is filled with possibilities & $\mathbf{0 . 8 1}$ & 0.17 \\
Many opportunities await me in the future & $\mathbf{0 . 7 7}$ & 0.13 \\
There is plenty of time left in my life to make new plans & $\mathbf{0 . 7 5}$ & 0.33 \\
Most of my life lies ahead of me & $\mathbf{0 . 6 5}$ & 0.20 \\
My future seems infinite to me & $\mathbf{0 . 5 6}$ & 0.56 \\
I could do anything I want in the future & $\mathbf{0 . 5 2}$ & 0.47 \\
As I get older I begin to experience time as limited & 0.04 & $\mathbf{0 . 8 5}$ \\
I have the sense that time is running out & 0.30 & $\mathbf{0 . 7 6}$ \\
There are only limited possibilities in my future & 0.31 & $\mathbf{0 . 7 2}$ \\
\hline
\end{tabular}

The highest loading for each item is shown in bold. A two-factor solution explained $62.08 \%$ of total variance and had an eigenvalue of 1.36 .

both subjective well-being measures, the CESD and PGCMS, whereas a focus on limitations was associated with the PGCMS and hair cortisol.

\section{Future Time Perspective as a Two-Dimensional Construct}

An exploratory factor analysis of the Future Time Perspective Scale with varimax rotation revealed a single-factor solution that explained $36 \%$ of the total variance and had an eigenvalue of 4.84. A two-factor solution however best fit the data and explained an additional $26 \%$ of the variance, with an eigenvalue of $1.36^{1}$. A confirmatory factor analysis resulted in a significant two-factor model as the raw data eigenvalue of 0.89 was greater than the permutation eigenvalue of 0.77 for the 95 th percentile [35]. In comparing our results to those of Cate and John [7], the same 7 items loaded onto the dimension a focus on opportunities with the same remaining 3 loading onto the dimension a focus on limitations. A focus on opportunities had a mean of $3.29(\mathrm{SD}=0.77$, range $=1.57-5.00)$ and held a Cronbach's alpha of 0.87 , while a focus on limitations had a mean of $3.02(\mathrm{SD}=0.85$, range $=1.00-5.00)$, Cronbach's alpha 0.78 . The correlation between these two dimensions was $0.49, \mathrm{p}<0.001$.

\footnotetext{
${ }^{1}$ In a separate data set $(\mathrm{n}=139$, mean age $=71.94, \mathrm{SD}=5.02)$ a two-factor solution of future time perspective also best described the data. The twofactor solution explained $63.53 \%$ of variance and held an eigenvalue of 1.57 . A confirmatory factor analysis resulted in a significant two-factor model as the raw data eigenvalue of 1.08 was greater than the permutation eigenvalue of 0.48 for the 95 th percentile.
}

Future Time Perspective and Well-Being
Table 2 lists the factor analysis loadings for each item of the Future Time Perspective Scale. Having replicated the two-dimensional structure of the Future Time Perspective Scale [7], we then created a meditation model for each dimension and tested the association each had with hair cortisol and subjective well-being measures the CESD and PGCMS.

\section{Associations between Future Time Perspective, \\ Subjective Well-Being and Hair Cortisol}

Our results revealed that a focus on opportunities was associated with both the PGCMS $[\mathrm{t}(65)=2.48, \mathrm{p}=0.02]$ and the CES-D [ $\mathrm{t}(65)=-2.21, \mathrm{p}=0.03$ ] but not with hair cortisol [ $\mathrm{t}(65)=0.30, \mathrm{p}=0.77]$. The overall mediation model was significant $\left[\mathrm{R}^{2}=0.33, \mathrm{~F}(65)=2.69, \mathrm{p}<0.01\right]$. After controlling for the indirect effects of the CES-D and PGCMS, the association between a focus on opportunities and hair cortisol was not significant $[\mathrm{t}(65)=0.19, \mathrm{p}=$ $0.85]$. Figure 1 provides for a visual summary of these results.

In contrast, a focus on limitations was not associated with either of the subjective well-being measures, the PGCMS $[\mathrm{t}(65)=1.45, \mathrm{p}=0.15]$ and the CES-D [ $\mathrm{t}(65)=$ $-0.16, \mathrm{p}=0.88]$, but was associated with hair cortisol $[\mathrm{t}(65)=-2.16, \mathrm{p}=0.03]$. The overall model was significant $\left[\mathrm{R}^{2}=0.33, \mathrm{~F}(9,56)=2.70, \mathrm{p}<0.01\right]$ and continued to be so when the PGCMS was not included $\left[\mathrm{R}^{2}=0.28\right.$, $\mathrm{F}(8,57)=2.37, \mathrm{p}=0.02]$. Controlling for the indirect effects of the CES-D and PGCMS revealed that the subjective well-being measures were mediating the association between a focus on limitations and hair cortisol $[\mathrm{t}(65)=$ 


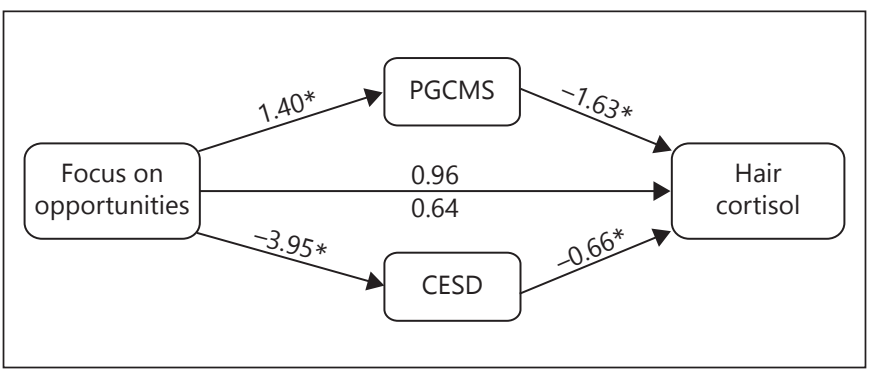

Fig. 1. A mediation model created through a bootstrapping approach $(n=66)$. In this model, a high focus on opportunities is associated with higher morale and less depressive symptoms. ${ }^{*} \mathrm{p}<$ 0.05 .

$-1.87, \mathrm{p}=0.07]$. Figure 2 provides a visual summary of these results.

Taken together and different from our initial prediction, only an open-ended future time perspective was associated with higher well-being. As hypothesized however, differential associations emerged for the two dimensions, a focus on opportunities was associated with both subjective well-being measures the CESD and PGCMS, whereas a focus on limitations was associated with hair cortisol.

\section{Discussion}

Both an open-ended future time perspective and a limited future time perspective have been associated with high well-being $[2,4,14,17]$. In an attempt to answer why a limited future time perspective can be associated with both high and low well-being, we first replicated the results of Cate and John [7] and demonstrated that the Future Time Perspective Scale [3] is composed of at least two different dimensions, a focus on opportunities and a focus on limitations. We then tested if these dimensions differentially associated with two different measures of subjective well-being, the CESD [11] and PGCMS [12] and a biological indicator of chronic stress, namely hair cortisol [10]. While both dimensions were related to wellbeing, each had unique associations. A high focus on opportunities was associated with higher morale and less depressive symptoms, whereas a low focus on limitations was associated with reduced hair cortisol. Though studies have shown these dimensions to be differentially associated with workplace variables $[8,9]$ this is the first study to demonstrate that each is independently associated with well-being in old age, and in particular, that these asso-

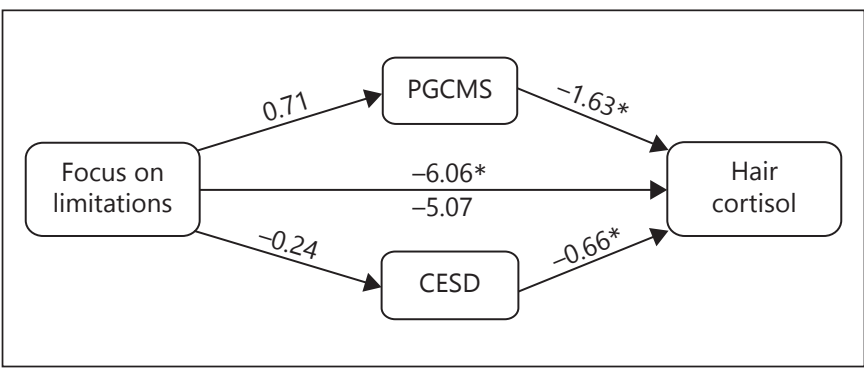

Fig. 2. A mediation model created through a bootstrapping approach $(n=66)$. In this model, a low focus on limitations is associated with less hair cortisol. This association however is mediated by the subjective well-being measures the PGCMS and CESD. $* \mathrm{p}<0.05$

ciations are dimension-specific to subjective and biological measures.

As a whole, our results align with the stream of studies documenting the benefits of an open-ended future time perspective $[2,17,18]$. However, we believe that further studies may consider the goals an individual sets as a potential underlying mechanism $[4,5]$. For example, an open-ended future time perspective might be associated with higher well-being if the individual has a goal that largely requires the passage of time, and hence personal limitations may matter less. Drawing from our older sample, perhaps one such goal is witnessing the high school graduation of a grandchild. In this case, goal completion inherently involves waiting for a particular outcome with potentially little personal control over goal completion. Alternatively, if an individual sets a goal that requires greater personal involvement or labor, perceived future limitations may be more salient. For instance, an individual might set a goal of improving their social relations, such as perhaps their relationship with their spouse. In this case, anticipated future limitations, such as declines in cognitive ability, limited physical capacity, or potential health problems, all may impact goal progress and in turn well-being. In both cases however, the goal an individual sets may or may not be in conflict with their future time perspective and as such may have the potential to be independently associated with well-being.

A recent meta-analysis done by Miller et al. [38] discussed the importance of measuring personal attributes to better understand why some events may be stressful to one individual and not another. Given our findings, future time perspective may help to offer a partial explanation. Speculatively, if one individual perceives remaining future time as open-ended, perhaps he or she experiences less stress when facing a potential setback in goal progress 
compared to an individual who perceives his/her remaining future time as more limited or scarce. More personally relevant and pertaining to the discussion above would be measuring the goals an individual sets in light of his/ her perception of remaining future time.

Further studies may also consider investigating whether or not the two dimensions of future time perspective differ depending on participant sex. Specifically, research documents that often in old age females take on the role of primary caregiver and thus may be especially concerned over not just their own personal limitations but also that of their partner's [39]. Given our findings that a focus on limitations is associated with hair cortisol, and a separate study showing that caregivers have greater hair cortisol concentration relative to non-caregivers, we believe a sex difference may be found [22].

In both the present study and that by Cate and John [7], the same 7 items of the Future Time Perspective Scale loaded onto the dimension a focus on opportunities with the remaining 3 loading onto the dimension a focus on limitations. We note that of the 10 items, only the negatively worded items loaded onto the dimension a focus on limitations. While the exact size of the item loadings differed between our study and that of Cate and John, we believe that this variance might be partially due to differing samples in that Cate and John [7] conducted their study on college-aged and midlife participants, whereas our participants had a mean age of 72 years. Regardless however, the evidence for the multi-dimensional nature of future time perspective seems strengthened given that both dimensions emerged across such different participant ages and demographics.

Relative to studies examining younger individuals, our sample of older adults had higher concentrations of hair cortisol [13]. Similar patterns between the young and old have been found in studies using salivary cortisol [40]. We speculate that this difference is attributable to changes in the HPA axis that occur with aging. Specifically, as we age there is greater wear and tear on the HPA axis such that a similar stressor in old age may elicit greater cortisol secretion than it would have at a younger age, in part because more time is required for an older individual to return to a pre-stressor baseline level [40].

The findings of this study have to be interpreted in light of several limitations. Our results are cross-sectional in nature, and as such we cannot draw temporal inferences. Future time perspective for instance may be associated with depressive symptoms, but the temporal order in which one may give rise to the other or if both come in tandem, cannot be answered. An individual might, for example, perceive future time as limited and with this acknowledge that certain goals are unlikely to be met. Alternatively, depression might change how an individual perceives future time such that they weigh more heavily on future limitations and obstacles. Though we applied a bootstrapping approach for our analyses, our sample size was not large enough to examine the key role of certain moderators such as the goals an individual may have. Since this study relied on hair cortisol, participants with little or no hair had to be excluded, resulting in a largely female sample. Given that hair cortisol accumulation may differ between males and females [13], future studies may consider whether the associations found in this study hold equally strong across sexes. An additional consideration would be to measure if participants have differential hair growth rates and if this influences cortisol accumulation. Lastly, all participants in the present study were volunteers, the vast majority (98.5\%) reported their health as being 'fair' to 'excellent' and only a quarter of participants $(28.8 \%)$ reported having provided care to someone within the last 3 months. Future studies may consider whether or not our results extend to those who are in different health conditions, stages in their career and have different time commitments.

\section{Conclusions}

Findings replicate and extend previous research by pointing to the two-dimensional nature of the Future Time Perspective Scale. Specifically, while both dimensions were associated with well-being, a high focus on opportunities was associated with higher morale and less depressive symptoms, whereas a low focus on limitations was associated with reduced hair cortisol. Future research may substantiate these findings by considering both dimensions of future time perspective in relation to the goals an individual sets, as well as by conducting a longitudinal research design through which temporal order of these associations may be better understood and established.

\section{Acknowledgements}

Christiane Hoppmann gratefully acknowledges the support of the Michael Smith Foundation for Health Research and the Canada Research Chairs Program. Pavel Kozik thanks his funding from the Natural Sciences and Engineering Research Council of Canada. We also wish to thank Clemens Kirschbaum for conducting the hair assays, without his contribution this project would not have come to fruition. 


\section{References}

1 Lewin K: Field theory and experiment in social psychology: concepts and methods. Am J Soc 1939;44:868-896.

2 Hicks JA, Trent J, Davis WE, King LA: Positive affect, meaning in life, and future time perspective: an application of socioemotional selectivity theory. Psychol Aging 2012;27: 181.

3 Carstensen LL, Lang FR (1996): Future Time Perspective Scale. Stanford, Stanford University, 1996.

$\checkmark 4$ Lang FR, Carstensen LL: Time counts: future time perspective, goals, and social relationships. Psychol Aging 2002;17:125-139.

$\checkmark 5$ Carstensen LL, Turan B, Scheibe S, Ram N, Ersner-Hershfield H, Samanez-Larkin GR, et al: Emotional experience improves with age: evidence based on over 10 years of experience sampling. Psychol Aging 2011;26:21-33.

6 Coudin G, Lima ML: Being well as time goes by: future time perspective and well-being. Int J Psychol Psychol Ther 2011;11: 219-232.

$\checkmark 7$ Cate RA, John OP: Testing models of the structure and development of future time perspective: maintaining a focus on opportunities in middle age. Psychol Aging 2007;22: 186-201.

$>8$ Zacher H, Frese M: Remaining time and opportunities at work: relationships between age, work characteristics, and occupational future time perspective. Psychol Aging 2009; 24:487-493.

9 Zacher H, Heusner S, Schmitz M, Zwierzanska MM, Frese M: Focus on opportunities as a mediator of the relationships between age, job complexity, and work performance. J Vocat Behav 2010;76:374-386.

$>10$ Kirschbaum C, Tietze A, Skoluda N, Dettenborn L: Hair as a retrospective calendar of cortisol production-increased cortisol incorporation into hair in the third trimester of pregnancy. Psychoneuroendocrinology 2009; 34:32-37.

11 Radloff LS: The CES-D scale a self-report depression scale for research in the general population. Appl Psychol Meas 1977;1:385-401.

$\checkmark 12$ Lawton MP: The Philadelphia geriatric center morale scale: a revision. J Gerontol 1975;30: 85-89.

-13 Dettenborn L, Tietze A, Kirschbaum C, Stalder T: The assessment of cortisol in human hair: associations with sociodemographic variables and potential confounders. Stress 2012; 15:578-588.

14 Fung HH, Carstensen LL, Lutz AM: Influence of time on social preferences: implications for life-span development. Psychol Aging 1999; 14:595-604
15 Fung HH, Carstensen LL: Goals change when life's fragility is primed: lessons learned from older adults, the September 11 attacks and SARS. Soc Cogn 2006;24:248-278.

16 Carstensen LL, Fredrickson BL: Influence of HIV status and age on cognitive representations of others. Health Psychol 1998;17:494503.

17 Allemand M, Hill PL, Ghaemmaghami P, Martin M: Forgivingness and subjective wellbeing in adulthood: the moderating role of future time perspective. J Res Pers 2012;46: 32-39.

18 Davis WE, Hicks JA: Maintaining hope at the 11th hour authenticity buffers the effect of limited time perspective on hope. Pers Soc Psychol Bull 2013;39:1634-1646.

19 Dettenborn L, Tietze A, Bruckner F, Kirschbaum C: Higher cortisol content in hair among long-term unemployed individuals compared to controls. Psychoneuroendocrinology 2010;35:1404-1409.

20 Piazza JR, Almeida DM, Dmitrieva NO, Klein LC: Frontiers in the use of biomarkers of health in research on stress and aging. J Gerontol B Psychol Sci Soc Sci 2010;65:513525.

21 Van Uum S, Sauve B, Fraser L, Morley-Forster P, Paul T, Koren G: Elevated content of cortisol in hair of patients with severe chronic pain: a novel biomarker for stress: short communication. Stress 2008;11:483-488.

22 Stalder T, Tietze A, Steudte S, Alexander N, Dettenborn L, Kirschbaum C: Elevated hair cortisol levels in chronically stressed dementia caregivers. Psychoneuroendocrinology 2014;47:26-30.

23 Skoluda N, Dettenborn L, Stalder T, Kirschbaum C: Elevated hair cortisol concentrations in endurance athletes. Psychoneuroendocrinology 2012;37:611-617.

24 Staufenbiel SM, Penninx BW, Spijker AT, Elzinga BM, van Rossum EF: Hair cortisol, stress exposure, and mental health in humans: a systematic review. Psychoneuroendocrinology 2013;38:1220-1235.

25 Baum A, Grunberg N: Measurement of stress hormones; in Cohen S, Kessler RC, Gordon LU (eds): Measuring Stress: A Guide for Health and Social Scientists. New York, Oxford University Press, 1995, pp 175-192.

26 Schwarz N: Self-reports: how the questions shape the answers. Am Psychol 1999;54:93105.

27 Cohen S, Janicki-Deverts D: Who's stressed? Distributions of psychological stress in the United States in probability samples from 1983, 2006, and 20091. J Appl Soc Psychol 2012;42:1320-1334.
28 Fichman HC, Fernandes CS, Nitrini R, Lourenço RA, de Paiva E, Cathery-Goulart M: Age and educational level effects on the performance of normal elderly on category verbal fluency tasks. Dement Neuropsychol 2009;3:49-54.

29 Haringsma R, Engels GI, Beekman AT, Spinhoven P: The criterion validity of the Center for Epidemiological Studies Depression Scale (CES-D) in a sample of self-referred elders with depressive symptomatology. Int J Geriatr Psychiatry 2004; 19:558-563.

30 Stalder T, Steudte S, Alexander N, Miller R, Gao W, Dettenborn L, et al: Cortisol in hair, body mass index and stress-related measures. Biol Psychol 2012;90:218-223.

31 Pereg D, Gow R, Mosseri M, Lishner M, Rieder M, Van Uum S, et al: Hair cortisol and the risk for acute myocardial infarction in adult men. Stress 2011;14:73-81.

32 Thomson S, Koren G, Fraser L-A, Rieder M, Friedman T, Van Uum S: Hair analysis provides a historical record of cortisol levels in Cushing's syndrome. Exp Clin Endocrinol Diabetes 2010;118:133-138.

33 Sauvé B, Koren G, Walsh G, Tokmakejian S, Van Uum SH: Measurement of cortisol in human hair as a biomarker of systemic exposure. Clin Invest Med 2007;30:E183-E191.

34 Kaiser HF: The application of electronic computers to factor analysis. Educ Psychol Meas 1960;20:141-151.

-35 O'Connor BP: SPSS and SAS programs for determining the number of components using parallel analysis and Velicer's MAP test. Behav Res Methods Instrum Comput 2000;32: 396-402.

>36 Preacher KJ, Hayes AF: Asymptotic and resampling strategies for assessing and comparing indirect effects in multiple mediator models. Behav Res Methods 2008;40:879-891.

37 Baron RM, Kenny DA: The moderator-mediator variable distinction in social psychological research: conceptual, strategic, and statistical considerations. J Pers Soc Psychol 1986;51:6.

38 Miller GE, Chen E, Zhou ES: If it goes up, must it come down? Chronic stress and the hypothalamic-pituitary-adrenocortical axis in humans. Psychol Bull 2007;133:25-45.

-39 Hoppmann CA, Gestorf D: Biobehavioral pathways underlying spousal health dynamics: its nature, correlates, and consequences. Gerontology 2014;60:458-465.

40 Nater UM, Hoppmann CA, Scott SB: Diurnal profiles of salivary cortisol and alpha-amylase change across the adult lifespan: evidence from repeated daily life assessments. Psychoneuroendocrinology 2013;38:3167-3171. 